\title{
A Case of Wilms Tumor with a Tumor Thrombus in a Boy with WAGR Syndrome
}

\author{
Soojung Lee, Hyo Jin Kim and In-sang Jeon \\ Department of Pediatrics, Gil Medical Center, Gachon University College of Medicine, Incheon, Korea
}

Intravascular extension of Wilms tumor (WT) can occur in approximately $4-10 \%$ of patients. In general, it does not cause any clinical problems because most of these tumors are small. Although there is no standard treatment currently, preoperative chemotherapy and delayed nephrectomy is generally recommended for children with WT accompanied by tumor thrombus. We report a rare case of WT, aniridia, genitourinary anomalies, and mental retardation (WAGR) syndrome in a boy who also had a huge inferior vena cava thrombus, $7 \mathrm{~cm}$ length. The prevalence of bilateral WT and tumor thrombus in WAGR has not been identified. The patient was successfully treated with neoadjuvant chemotherapy to decrease the size of the tumor thrombus with WT and delayed nephrectomy following chemotherapy without any invasive intervention and did not show complications.

Key Words: WAGR syndrome, Wilms tumor, Tumor thrombus, Inferior vena cava
pISSN 2233-5250 / eISSN 2233-4580 https://doi.org/10.15264/cpho.2020.27.2.134 Clin Pediatr Hematol Oncol 2020;27:134 137

Received on April 21, 2020 Revised on May 21, 2020 Accepted on June 19, 2020

Corresponding Author: In-sang Jeon Department of Pediatrics, Gachon University Gil Medical Center, 14 Namdongdaero 774 beongil, Namdong-gu, Incheon 21565 , Korea

Tel: $+82-32-458-2774$

Fax: +82-32-460-3224

E-mail: isjeon@gilhospital.com ORCID ID: orcid.org/0000-0001-8714-9403

\section{Introduction}

Wilms tumor (WT), aniridia, genitourinary anomalies, and mental retardation (WAGR) syndrome is a rare genetic disease caused by a continuous chromosome deletion at $11 \mathrm{p} 13$, which results in the loss of two important genes (WT1 and PAXG) associated with WT and aniridia, respectively $[1,2]$.

WT tends to invade blood vessels in the form of a tumor thrombus. The incidence of intravascular extension with WT into the inferior vena cava has been reported $4 \%$ to $10 \%$ patients. Extension of tumor thrombus into the renal vein has been reported more common (59-85\%) and into the right atrium is less reported (0.7-1\%) [3]. Previous studies have recommended preoperative chemotherapy for tumor thrombus with WT, which effec- tively leads to tumor shrinkage and venous tumor thrombus regression [3-5]. Primary surgical treatment should be performed after considering several factors such as age at diagnosis, size, location, and extent of the thrombus, surgical accessibility, clinical symptoms, and the high risk of pulmonary embolism $[3,4,6]$. But, recently preoperative chemotherapy is recommended for all WT patients except when the patient's vital signs are unstable $[4,5]$.

The present report describes a presentation of WAGR syndrome in a patient who also had a huge IVC tumor thrombus. WT and tumor thrombus were successfully treated with neoadjuvant chemotherapy and delayed nephrectomy without any requirement for invasive procedures and complications. 


\section{Case Report}

The patient was born after 40 gestational weeks with a birth weight of $2,860 \mathrm{~g}$ ( $15^{\text {th }}$ percentile). He was the third-born child, with no specific medical history during the perinatal period. He was diagnosed with bilateral congenital aniridia at 80 days of age. Bilateral cryptorchidism and malrotation of the left kidney were identified on abdominal ultrasonography at 4 month of age. In addition, chromosome analysis test detected a deletion of the chromosome 11p11.2-p13 region (Fig. 1A). Thus, he was diagnosed with WAGR syndrome.

We planned routine screening abdominal ultrasonography and physical examinations to evaluate the progression of the malrotation of the left kidney and to confirm appearing WT. However, the patient was lost to follow-up. Abdominal ultrasonography was performed during hospitalization with the flu at the age of 1 years and 7 months, and it revealed no significant change of both kidneys. The patient was recommended to routine checkup, but he did not visit the hospital subsequently.

He was admitted to the emergency room at the age of 2 years and 10 months due to abdominal pain and abdominal distension. In the right flank, a huge abdominal mass was palpable. Abdominopelvic computed tomog- raphy (CT) showed bilateral renal masses, suggestive of WT. A heterogeneously enhancing mass measuring $12 \times$ $8 \times 9 \mathrm{~cm}$ was present in the right kidney and two hypoenhancing masses measuring approximately $0.7 \mathrm{~cm}$ and $1.3 \mathrm{~cm}$ in diameter with irregular margins were present in the mid-portion and the upper portion of the left kidney. Intravascular and liver metastases were identified on abdominopelvic CT. A tumor thrombus, approximately $7 \mathrm{~cm}$ in length, was present in the intra-hepatic and infra-hepatic IVC (Fig. 1B).

Urinalysis showed hematuria with 21-30 red blood cells (RBCs) per high-power field. Dysmorphic RBCs were not counted. On developmental evaluation, the patient also showed delays in language development and motor skills. On ophthalmic examination, he showed nystagmus and strabismus. He also had aniridia and macular aplasia in both eyes.

The patient received preoperative chemotherapy with vincristine, dactinomycin, and doxorubicin. After receiving four cycles of preoperative chemotherapy, the size of the mass in the right kidney was decreased about $8 \times 5 \times$ $6 \mathrm{~cm}$ and the regression of IVC thrombus observed. However, there was no change in the two masses in the left kidney, they were considered non-malignant masses. Right nephrectomy was performed after four cycles of preoperative chemotherapy over 12 weeks. Histological
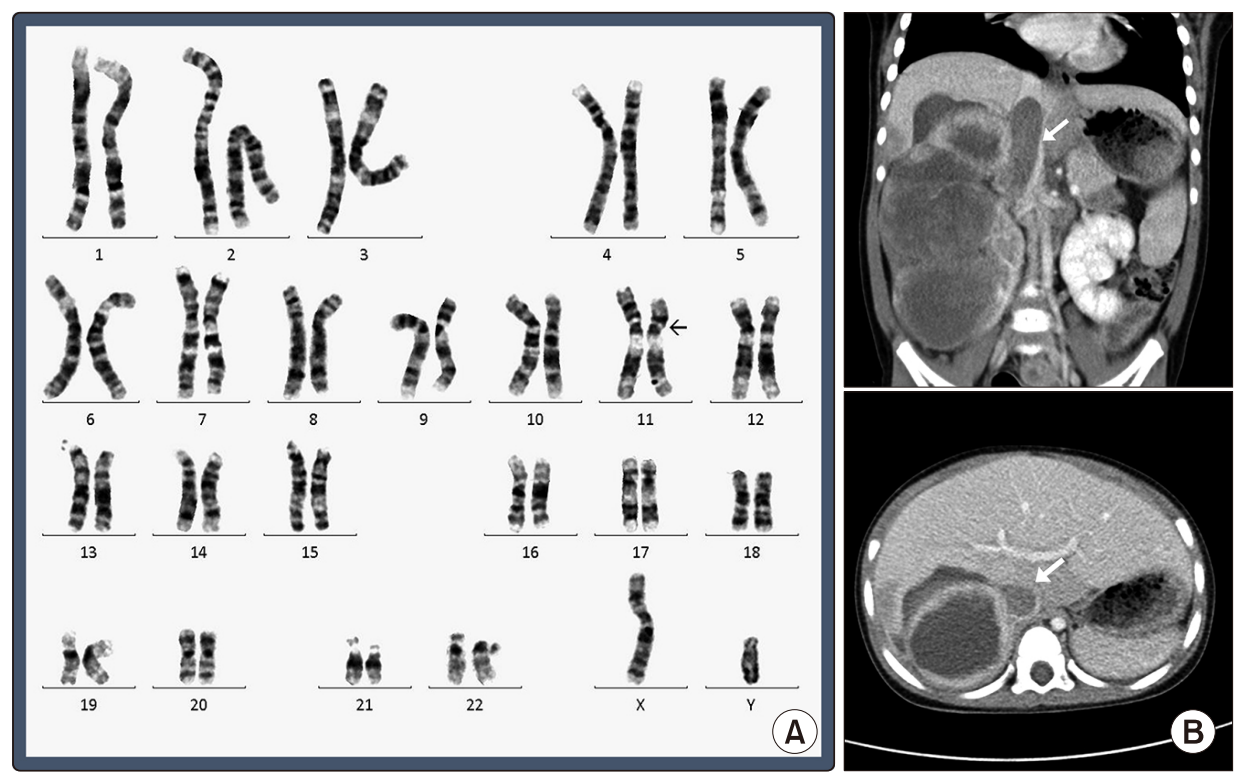

Fig. 1. (A) Chromosome analysis using GTL-banding at the level of 400-550 bands. The karyotype was 46,XY,del(11)(p11.2-p13). (B) Abdominal computed tomography scans showing a heterogeneously enhancing mass of size $12 \times 8 \times 9 \mathrm{~cm}$ in the right kidney and tumor thrombus, approximately $7 \mathrm{~cm}$ length in the intra-hepatic and infra-hepatic inferior vena cava (white arrow). 

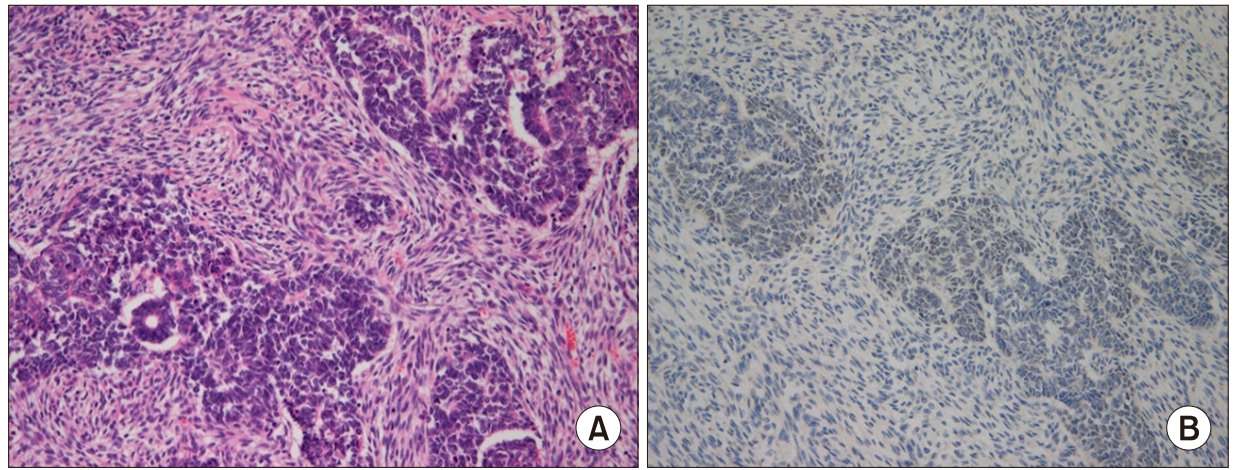

Fig. 2. (A) Light microscopy finding showing favorable histology, with blastermal, stromal, and epithelial elements (hematoxylin and eosin staining, $\times 200$ ). (B) Immunostaining with WT1 finding showing negative (WT1 staining, $\times 200$ ).

evaluation of the right kidney revealed favorable triphasic histological features of WT (Fig. 2A). Immunohistochemical staining was negative for the Wilms tumor gene product, WT1 (Fig. 2B).

The patient received nine cycles postoperative chemotherapy with radiotherapy. After two cycles of postoperative chemotherapy, left partial nephrectomy was performed. The histopathological result of the two masses from the left kidney revealed disorganized renal parenchyma and variable-sized cysts with inflammatory cell infiltration.

He received additional seven cycles of postoperative chemotherapy over 19 weeks. After postoperative chemotherapy, no residual WT and nearly complete regression of IVC tumor thrombus was detected on abdominopelvic CT.

\section{Discussion}

WAGR syndrome is diagnosed based on characteristic clinical features and genetic analysis WT is identified in $30-45 \%$ of the patients diagnosed with WAGR syndrome $[1,2]$. Although WT was not confirmed at the initial diagnosis, it could be found at a later follow-up like in the present case. The increased possibility of late-appearing WT has been reported in some cases of WAGR syndrome $[7,8]$. Therefore, imaging studies and physical examination are required through periodic outpatient follow-up observations.

The survival rate of patients with WT is greater than 85-90\% [9,10]. WT may also be accompanied by adjacent vascular invasion (renal veins, the IVC, and the right at- rium) [3]. WT patients with intravascular extension have similar or slightly worse overall survival than those without intravascular extension [5]. A diagnosis is usually made before surgery using imaging examinations. Symptoms of tumor thrombus vary in severity, but most patients are asymptomatic [5,11].

The treatment plan of WT is determined by multiple factors including patients' age at the time of diagnosis, tumor stage, histological response to therapy, and results of the chromosome study $[9,10]$. Intravascular extension of WT is also an important factor to consider while choosing the treatment. Traditionally, primary surgical treatment of the tumor and the thrombus has been perfomed [10]. However, the incidence of surgical complications has been reported in intravascular extension of WT $[4,5,11]$.

Lall et al. compared the outcome of immediate nephrectomy with that of preoperative chemotherapy and delayed nephrectomy in patients with WT and intravascular invasion [5]. Fifty-nine of the 730 children with WT were diagnosed with intravascular extension. Among the 52 patients who received preoperative chemotherapy, significant shrinkage of the tumor and the thrombus was demonstrated in 35 (67\%) patients. Sixteen of 59 (27\%) children died as they had unfavorable histology or complications. Surgical complications such as uncontrollable hemorrhage and other complications such as pulmonary embolism, severe respiratory distress, and tumor recurrence or progression were observed in these patients. Similarly, prior studies reported neoadjuvant chemotherapy causes tumor regression in 30-73\% [4]. Now, both Society of International Pediatric Oncology and 
Children's oncology group recommend preoperative chemotherapy and delayed surgical resection to reduce the risk of surgical morbidity and mortality $[4,5]$.

However, primary surgery would be indicated in patients with unstable vital signs due to the thrombus, which might dislocate and cause acute symptoms. Several surgical interventions such as IVC ligation, segmental IVC resection and reconstruction, and IVC filter placement are necessary in patients with tumors involving more than half of the circumference of the IVC, in cases of complete chronic IVC obstruction in the absence of clinical symptoms of venostasis, and in cases of a high risk of postoperative pulmonary embolisms [6].

In conclusion, clinicians should pay attention to changes in the clinical conditions for early diagnosis and treatment of WAGR syndrome. Moreover, all children with WT should be carefully evaluated using various imaging modalities to avoid underdiagnosis of intravascular tumor extension. Preoperative chemotherapy and delayed nephrectomy for huge IVC thrombus with WT could be recommended even in the case of WAGR syndrome.

\section{Conflict of Interest Statement}

The authors have no conflict of interest to declare.

\section{References}

1. Fischbach BV, Trout KL, Lewis J, Luis CA, Sika M. WAGR syndrome: a clinical review of 54 cases. Pediatrics 2005;116: 984-8.

2. Muto R, Yamamori S, Ohashi H, Osawa M. Prediction by FISH analysis of the occurrence of Wilms tumor in aniridia patients. Am J Med Genet 2002;108:285-9.

3. Al Diab A, Hirmas N, Almousa A, et al. Inferior vena cava involvement in children with Wilms tumor. Pediatr Surg Int 2017;33:569-73.

4. Emir S. Wilms tumor with intravascular tumor thrombus. Transl Pediatr 2014;3:29-33.

5. Lall A, Pritchard-Jones K, Walker J, et al. Wilms' tumor with intracaval thrombus in the UK Children's Cancer Study Group UKW3 trial. J Pediatr Surg 2006;41:382-7.

6. Ayyathurai R, Garcia-Roig M, Gorin MA, et al. Bland thrombus association with tumour thrombus in renal cell carcinoma: analysis of surgical significance and role of inferior vena caval interruption. BJU Int 2012;110:E449-55.

7. Clericuzio CL. Clinical phenotypes and Wilms tumor. Med Pediatr Oncol 1993;21:182-7.

8. Clericuzio CL. Recognition and management of childhood cancer syndromes: a systems approach. Am J Med Genet 1999; 89:81-90.

9. Aldrink JH, Heaton TE, Dasgupta R, et al. Update on Wilms tumor. J Pediatr Surg 2019;54:390-7.

10. Davidoff AM. Wilms' tumor. Curr Opin Pediatr 2009;21:35764 .

11. Shamberger RC, Ritchey ML, Haase GM, et al. Intravascular extension of Wilms tumor. Ann Surg 2001;234:116-21. 